Undziakiewicz Adrian, Sekuła Michał, Smoluchowski Krzysztof, Sokół Dorota, Świerczyńska Blanka, Piecewicz-Szczęsna Halina. The use of lung ultrasound in the diagnosis of pneumothorax in trauma patients. Journal of Education, Health and Sport. 2020;10(9):332-337. eISSN 2391-8306. DOI http://dx.doi.org/10.12775/JEHS.2020.10.09.037

https://apcz.umk.pl/czasopisma/index.php/JEHS/article/view/JEHS.2020.10.09.037

https://zenodo.org/record/4029663

The journal has had 5 points in Ministry of Science and Higher Education parametric evaluation. § 8. 2) and § 12. 1. 2) 22.02.2019.

This article is published with open access at Licensee Open Journal Systems of Nicolaus Copernicus University in Torun, Poland Open Access. This article is distributed under the terms of the Creative Commons Attribution Noncommercial License which permits any noncommercial use, distribution, and reproduction in any medium, provided the original author (s) and source are credited. This is an open access article licensed under the terms of the Creative Commons Attribution Non commercial license Share alike. (http://creativecommons.org/licenses/by-nc-sa/4.0/) which permits unrestricted, non commercial use, distribution and reproduction in any medium, provided the work is properly cited. The authors declare that there is no conflict of interests regarding the publication of this paper.

Received: 20.08.2020. Revised: 25.08.2020. Accepted: 15.09.2020.

\title{
The use of lung ultrasound in the diagnosis of pneumothorax in trauma patients
}

\author{
Adrian Undziakiewicz ${ }^{1}$, Michał Sekuła ${ }^{1}$, Krzysztof Smoluchowski ${ }^{1}$, Dorota Sokół ${ }^{1}$, \\ Blanka Świerczyńska ${ }^{1}$, Halina Piecewicz-Szczęsna ${ }^{2}$ \\ ${ }^{1}$ Student Research Circle at the Department of Epidemiology and Clinical Research Methodology, Medical \\ University of Lublin, Poland \\ ${ }^{2}$ Department of Epidemiology and Clinical Research Methodology, Medical University of Lublin, Poland
}

Adrian Undziakiewicz, https://orcid.org/0000-0002-1191-1366;

adrian.undziakiewicz@gmail.com (corresponding author)

Michał Sekuła, https://orcid.org/0000-0001-8378-9964; michalsekula061996@ gmail.com

Krzysztof Smoluchowski, https://orcid.org/0000-0001-9237-3346;

ksmoluchowski@gmail.com

Dorota Sokó1, https://orcid.org/0000-0003-2059-7951; dorota.soko19606@gmail.com

Blanka Świerczyńska, https://orcid.org/0000-0001-8782-8625;

swierczynska.blanka@gmail.com

Halina Piecewicz-Szczęsna, https://orcid.org/0000-0002-0573-7226; halpiec@wp.pl 


\begin{abstract}
Introduction and purpose: Pneumothorax is a condition in which air is present in the pleural cavity and often has a traumatic etiology. It can be a life-threatening condition, making quick and effective diagnosis essential. Chest ultrasound can be a useful study for this purpose.
\end{abstract}

A brief description of the state of knowledge: The sonographic signs of pneumothorax are absence of lung sliding, absence of B-lines, absence of lung pulse and presence of lung point. When a pneumothorax is suspected, the supine position is the most appropriate position for the patient. The sonographic technique consists of exploration of the least gravitationally dependent areas progressing more laterally. The bedside sonographic diagnosis of pneumothorax can be performed with most ultrasound machines without the need of any sophisticated functions. The average time to perform this examination varies from two to three minutes. In the most recent meta-analysis chest ultrasound sensitivity and specificity were 0.91 (95\% CI: 0.85 to 0.94 ) and 0.99 (95\% CI: 0.97 to 1.00), respectively. There was a significant difference in the sensitivity of CUS compared to CXR with an absolute difference in sensitivity of 0.44 (95\% CI: 0.27 to $0.61 ; \mathrm{P}<0.001)$.

Conclusions: Chest ultrasound is a safe, effective and sensitive method of diagnosing pneumothorax. It may be considered as the first study of trauma patients with suspected pneumothorax.

Key words: Pneumothorax; chest ultrasound; ultrasonography

\title{
1. Introduction and purpose
}

Pneumothorax is a condition in which air is present in the pleural cavity. This is the second most common consequence of a blunt chest injury ${ }^{1}$. It can quickly turn into a life-threatening condition that requires a quick response and adequate supply to avoid consequences that are dangerous for the patient ${ }^{1,2}$. Therefore, diagnosis as soon as possible is crucial. Traditionally, the first examination used is chest X-ray, but especially when performed in the supine position, it is characterized by low sensitivity ${ }^{3-5}$. This creates a problem because a large proportion of trauma patients are in the supine position. The gold standard in the detection of pneumothorax is computed tomography, which is able to visualize the occult pneumothorax, i.e. one that is not visible on a chest X-ray ${ }^{6,7}$. However, it also has limitations such as the dose of radiation taken by the patient, the cost, and the fact that some patients are not transportable for examination. Chest ultrasound is a study that has become more and more popular in intensive care units in recent years. It is non-invasive, portable and does not generate radiation. It is performed in a supine position and is accessible at the bedside, therefore it does not require transport and can be safely performed even during resuscitation. It is characterized by high sensitivity and specificity, which allows for an effective rule in and rule out the diagnosis of pneumothorax ${ }^{4,8-10}$. 


\section{Description of the state of knowledge}

The sonographic signs of pneumothorax are absence of lung sliding, absence of B-lines, absence of lung pulse and presence of lung point ${ }^{10}$. Lung sliding is the movement of the pleural line synchronous with tidal ventilation visualized in $2 \mathrm{D}^{11}$. When present, it means that the parietal and visceral pleura are in touch and regional ventilation is present, thus excluding pneumothorax ${ }^{11,12}$. Absence of lung sliding suggests pneumothorax with context-dependent accuracy ${ }^{11,13}$. However, it does not necessarily indicate a pneumothorax ${ }^{14}$.

B-lines are vertical hyperechoic comet-tail artifacts deriving from the pleural line and moving synchronously with it. Their presence makes it possible to rule out pneumothorax ${ }^{15}$.

The lung pulse refers to the subtle rhythmic movement of the visceral upon the parietal pleura with cardiac oscillations ${ }^{10}$. Its presence indicates that the parietal and visceral pleura are in touch but regional ventilation is impaired. It confirms absence of regional ventilation and rules out pneumothorax at the same time ${ }^{16}$.

The described signs play a greater role in exclusion than in confirming pneumothorax, since their absence is not specific to pneumothorax. The presence of any of them makes it possible to rule out pneumothorax in a particular area of insonation. On the other hand, a symptom specific for pneumothorax is lung point ${ }^{17}$. It is the contact point between the collapsed lung and the pneumothorax air collection and is described as alternation of normal and abolished sliding during tidal ventilation ${ }^{11}$. Its presence confirms pneumothorax with a sensitivity of $66 \%$ and a specificity of $100 \%^{18}$. A lung point will be absent in complete pneumothorax where the lung is completely retracted to the hilum ${ }^{5}$. The location of the lung point may be used to provide a semi-quantitative estimate of the size of a pneumothorax ${ }^{19}$. In extreme emergency, absence of any movement of the pleural line, either horizontal (sliding) or vertical (pulse), coupled with absence of B-lines allows prompt and safe diagnosis of pneumothorax without the need for searching the lung point $t^{10}$.

When a pneumothorax is suspected, the most appropriate position for lung ultrasound is supine because pleural air collects in the least dependent part of the thorax, or the anterior chest wall, which is highly accessible to ultrasound imaging ${ }^{20}$. According to the recommendations, in the supine patient, the sonographic technique consists of exploration of the least gravitationally dependent areas progressing more laterally. In most patients without pre-existing pleural diseases, the pneumothorax will be located in the least of the gravitationally dependent areas of the pleurac cavities. The bedside sonographic diagnosis of pneumothorax can be performed with most ultrasound machines without the need of any sophisticated functions ${ }^{21}$. During assessment for pneumothorax in adults, a microconvex probe is preferred. However, other transducer (e.g., linear array, phased array, convex) may be chosen based on physician preference and clinical setting ${ }^{3,10,22}$. High-frequency linear probe allows for higher resolution which translates into better visualization of superficial structures and the pleural line. A microconvex or curvilinear probe may be more suitable for deeper lung imaging as it provides better penetration. In cases where different diagnoses are taken into account and there is a need for a "whole-body" examination, the microconvex probe should be considered as the first choice ${ }^{21,23}$. Ultimately, the choice of transducer depends on the experience and preferences of the examiner. 
The probe should be placed on anterior chest wall in sagittal orientation to allow visualisation of at least two ribs and the pleural line in between them. Then, following from medial to lateral, visualize the pleural line in 2nd-4th intercostal spaces, as this is where most of the pneumothoraxes will be visible. When in doubt, comparing images from the opposite site may facilitate diagnosis. M-Mode and Power Doppler also can be used ${ }^{10,21,23}$. The average time to perform this examination varies from two to three minutes ${ }^{24,25}$.

In the most recent meta-analysis by Chan et al., which aimed to compare the effectiveness of the diagnosis of chest ultrasonography by frontline non-radiologist physicians versus supine chest $\mathrm{x}$-ray for diagnosis of pneumothorax in trauma patients in the emergency department, ultrasonography had a sensitivity of 0.91 (95\% CI: 0.85 to 0.94$)$ and a specificity of 0.99 (95\% CI: 0.97 to 1.00 ). In the same study, chest radiography had a sensitivity of 0.47 (95\% CI: 0.31 to 0.63 ) and a specificity of 1.00 (95\% CI: 0.97 to 1.00 ). This study included 13 prospective, paired comparative accuracy studies in which patients were suspected of having pneumothorax. There was a significant difference in the sensitivity of CUS compared to CXR with an absolute difference in sensitivity of 0.44 (95\% CI: 0.27 to $0.61 ; \mathrm{P}<0.001)$. The results of this meta-analysis show that the diagnostic accuracy of chest ultrasonography performed by frontline non-radiologist physicians was superior to supine CXR for diagnostic traumatic pneumothorax in trauma patients ${ }^{4}$.

Staub et al.'s study included 14 studies in the primary analysis. This meta-analysis was designed to evaluate the effectiveness of chest ultrasonography in emergency diagnosis of traumatic pneumothorax in adult patients. Studies included patients with different types of trauma (multiple trauma, blunt or penetrating chest trauma, blunt or penetrating torso trauma). All the studies used chest computed tomography as the reference standard. Reported experience and training differed from study to study. Chest ultrasonography had AUC of 0,979 for the diagnosis of traumatic pneumothorax (sensitivity $95 \%$ CI: $0.75-0.90$, specificity 95\% CI: 0.97-0.99). This study shows that lung ultrasound has good sensitivity in detecting pneumothorax in trauma patients and can be a good diagnostic tool ${ }^{26}$.

Another meta-analysis compared the effectiveness of chest ultrasonography with that of a chest radiography. 19 studies were included in the subgroup of trauma patients. The pooled sensitivity and specificity for chest ultrasound in this subgroup were 0.85 (95\% CI: 0.78-0.91) and 0.99 (95\% CI: 0.99-1.00), respectively. In the same subgroup, the sensitivity and specificity for chest radiography were 0.46 (95\% CI: 0.35-0.57) and 0.99 (95\% CI: 0.960.99), respectively. Additionally, a meta-regression was performed which showed that the sensitivity and specificity of the ultrasound was higher when performed by the emergency physician (0.88 (95\% CI: 0.78-0.91) and 0.99 (95\% CI: 0.98-1.00) vs 0.81 (95\% CI: $0.73-$ $0.90)$ and 0.98 (95\% CI: $0.96-0.99))^{9}$.

\section{Conclusions}

Chest ultrasound is a sensitive method of diagnosing pneumothorax. It can be successfully used for this purpose in patients with chest injuries. It is a more precise method than chest radiography and should be considered as an initial diagnostic strategy in trauma patients with suspected pneumothorax. 


\section{List of references}

1. Wilson H, Ellsmere J, Tallon J, Kirkpatrick A. Occult pneumothorax in the blunt trauma patient: Tube thoracostomy or observation? Injury. 2009;40(9):928-931. doi:10.1016/j.injury.2009.04.005

2. Noppen M, De Keukeleire T. Pneumothorax. Respiration. 2008;76(2):121-127. doi:10.1159/000135932

3. Ball CG, Kirkpatrick AW, Laupland KB, et al. Factors related to the failure of radiographic recognition of occult posttraumatic pneumothoraces. Am J Surg. 2005;189(5 SPEC. ISS.):541-546. doi:10.1016/j.amjsurg.2005.01.018

4. Chan KK, Joo DA, McRae AD, et al. Chest ultrasonography versus supine chest radiography for diagnosis of pneumothorax in trauma patients in the emergency department. Cochrane Database Syst Rev. 2020;2020(7). doi:10.1002/14651858.CD013031.pub2

5. Mayo PH, Copetti R, Feller-Kopman D, et al. Thoracic ultrasonography: a narrative review. Intensive Care Med. 2019;45(9):1200-1211. doi:10.1007/s00134-019-05725-8

6. Ball CG, Hameed SM, Evans D, Kortbeek JB, Kirkpatrick AW. Occult pneumothorax in the mechanically ventilated trauma patient. Can J Surg. 2003;46(5):373-379. Accessed August 27, 2020. /pmc/articles/PMC3211710/?report=abstract

7. Trupka A, Waydhas C, Hallfeldt KKJ, Nast-Kolb D, Pfeifer KJ, Schweiberer L. Value of thoracic computed tomography in the first assessment of severely injured patients with blunt chest trauma: Results of a prospective study. J Trauma - Inj Infect Crit Care. 1997;43(3):405-411. doi:10.1097/00005373-199709000-00003

8. Abdalla W, Elgendy M, Abdelaziz AA, Ammar MA. Lung ultrasound versus chest radiography for the diagnosis of pneumothorax in critically ill patients: A prospective, single-blind study. Saudi J Anaesth. 2016;10(3):265-269. doi:10.4103/1658-354X.174906

9. Ebrahimi A, Yousefifard M, Kazemi HM, et al. Diagnostic accuracy of chest ultrasonography versus chest radiography for identification of pneumothorax: A systematic review and meta-analysis. Tanaffos. 2014;13(4):29-40. Accessed August 21, 2020. /pmc/articles/PMC4386013/?report=abstract

10. Volpicelli G, Elbarbary M, Blaivas M, et al. International evidence-based recommendations for point-of-care lung ultrasound. In: Intensive Care Medicine. Vol 38. Springer; 2012:577-591. doi:10.1007/s00134-012-2513-4

11. Mojoli F, Bouhemad B, Mongodi S, Lichtenstein D. Lung ultrasound for critically ill patients. Am J Respir Crit Care Med. 2019;199(6):701-714. doi:10.1164/rccm.2018020236CI

12. Lichtenstein DA, Menu Y. A bedside ultrasound sign ruling out pneumothorax in the critically ill: Lung sliding. Chest. 1995;108(5):1345-1348. doi:10.1378/chest.108.5.1345

13. Blaivas M, Lyon M, Duggal S. A prospective comparison of supine chest radiography and bedside ultrasound for the diagnosis of traumatic pneumothorax. Acad Emerg Med. 2005;12(9):844-849. doi:10.1197/j.aem.2005.05.005

14. Golub J, Markota A, Stožer A, et al. Absence of lung sliding is not a reliable indicator of pneumothorax in patients who require high PEEP. Crit Care. 2015;19(Suppl 1):P139. doi:10.1186/cc14219 
15. Lichtenstein D, Mezière G, Biderman P, Gepner A. The comet-tail artifact: An ultrasound sign ruling out pneumothorax. Intensive Care Med. 1999;25(4):383-388. doi: $10.1007 / \mathrm{s} 001340050862$

16. Lichtenstein DA, Lascols N, Prin S, Mezière G. The "lung pulse": An early ultrasound sign of complete atelectasis. Intensive Care Med. 2003;29(12):2187-2192. doi:10.1007/s00134-003-1930-9

17. Lichtenstein D. Novel approaches to ultrasonography of the lung and pleural space: Where are we now? Breathe. 2017;13(2):100-111. doi:10.1183/20734735.004717

18. Lichtenstein D, Mezière G, Biderman P, Gepner A. The "lung point": An ultrasound sign specific to pneumothorax. Intensive Care Med. 2000;26(10):1434-1440. doi:10.1007/s001340000627

19. Volpicelli G, Boero E, Sverzellati N, et al. Semi-quantification of pneumothorax volume by lung ultrasound. Intensive Care Med. 2014;40(10):1460-1467. doi:10.1007/s00134-014-3402-9

20. Kruisselbrink R, Chan V, Cibinel GA, Abrahamson S, Goffi A. I-AIM (Indication, Acquisition, Interpretation, Medical Decision-making) Framework for Point of Care Lung Ultrasound. Anesthesiology. 2017;127(3):568-582. doi:10.1097/ALN.0000000000001779

21. Husain LF, Hagopian L, Wayman D, Baker WE, Carmody KA. Sonographic diagnosis of pneumothorax. In: Journal of Emergencies, Trauma and Shock. Vol 5. Wolters Kluwer - Medknow Publications; 2012:76-81. doi:10.4103/0974-2700.93116

22. Tocino IM, Miller MH, Fairfax WR. Distribution of pneumothorax in the supine and semirecumbent critically ill adult. Am J Roentgenol. 1985;144(5):901-905. doi:10.2214/ajr.144.5.901

23. Goffi A, Kruisselbrink R, Volpicelli G. The sound of air: point-of-care lung ultrasound in perioperative medicine. Can J Anesth. 2018;65(4):399-416. doi:10.1007/s12630-0181062-x

24. Dulchavsky SA, Schwarz KL, Kirkpatrick AW, et al. Prospective evaluation of thoracic ultrasound in the detection of pneumothorax. In: Journal of Trauma - Injury, Infection and Critical Care. Vol 50. Lippincott Williams and Wilkins; 2001:201-205. doi:10.1097/00005373-200102000-00003

25. Kirkpatrick AW, Sirois M, Laupland KB, et al. Hand-held thoracic sonography for detecting post-traumatic pneumothoraces: The extended focused assessment with sonography for trauma (EFAST). J Trauma - Inj Infect Crit Care. 2004;57(2):288-295. doi:10.1097/01.TA.0000133565.88871.E4

26. Staub LJ, Biscaro RRM, Kaszubowski E, Maurici R. Chest ultrasonography for the emergency diagnosis of traumatic pneumothorax and haemothorax: A systematic review and meta-analysis. Injury. 2018;49(3):457-466. doi:10.1016/j.injury.2018.01.033 